\title{
Excitonic BCS-BEC crossover at finite temperature: Effects of repulsion and electron-hole mass difference
}

\author{
Yuh Tomio, Kotaro Honda, and Tetsuo Ogawa \\ CREST, JST, and Department of Physics, Osaka University, Toyonaka, Osaka 560-0043, Japan
}

(Dated: September 6, 2018)

\begin{abstract}
The BCS to Bose-Einstein condensation (BEC) crossover of electron-hole $(e-h)$ pairs in optically excited semiconductors is studied using the two-band Hubbard model with both repulsive and attractive interactions. Applying the self-consistent $t$-matrix approximation combined with a local approximation, we examine the properties of a normal phase and an excitonic instability. The transition temperature from the normal phase to an $e-h$ pair condensed one is studied to clarify the crossover from an $e$ - $h$ BCS-like state to an excitonic Bose-Einstein condensation, which takes place on increasing the $e$ - $h$ attraction strength. To investigate effects of the repulsive interaction and the $e$ - $h$ mass difference, we calculate the transition temperature for various parameters of the interaction strengths, the $e-h$ particle density, and the mass difference. While the transition temperature in the $e$ - $h$ BCS regime is sufficiently suppressed by the repulsive interaction, that of the excitonic BEC is largely insensitive to it. We also show quantitatively that in the whole regime the mass difference leads to large suppression of the transition temperature.
\end{abstract}

PACS numbers: 71.10.Hf, 71.35.Lk, 03.75.Nt

\section{INTRODUCTION}

The Bose-Einstein condensation (BEC) of excitons in solids is one of the most interesting subjects in condensed matter physics $\frac{1}{\underline{1}}$ In this phenomenon the attractive Coulomb interaction between electrons and holes plays an essential role in forming bosonic electron-hole $(e-h)$ bound pairs. This interaction and the bosonic nature strongly affect the statistical and thermodynamic properties of $e-h$ systems.

In $e-h$ systems realized in photoexcited semiconductors, therefore, various remarkable properties are expected to depend on $e-h$ density, temperature, etc., and they have been investigated extensively both experimentally and theoretically..3 In particular, metal-insulator transitions have attracted interest for many years: One is the exciton Mott transition from an exciton or biexciton insulating gas phase to an $e$ $h$ plasma (normal) metallic phase with increasing $e-h$ density. Another, which is our main target in this work, is the transition from the normal phase to an $e-h$ pair condensed one. At low $e-h$ density, strongly bound $e-h$ pairs are expected to undergo BEC as an exciton gas at cryogenic temperatures ${ }^{4.5}$ On the other hand, at high $e-h$ density where the mean interparticle distance is shorter than the exciton Bohr radius, weakly bound $e-h$ pairs should behave like the Cooper pairs in conventional superconductors at sufficiently low temperatures, that is, the Bardeen-Cooper-Schrieffer (BCS) state of $e-h$ pairs ${ }^{6.7}$ There have been many experimental attempts to observe such a coherent condensation of $e-h$ bound pairs in solids such as $\mathrm{Cu}_{2} \mathrm{O}$, $\mathrm{CuCl}$ and $\mathrm{GaAs}{ }^{8.9 .10 .11 .12}$ At present, however, definitive evidence is still lacking in experiments.

Since the $e-h$ recombination is usually much slower than the intraband relaxation, we focus on a quasi-thermal-equilibrium state of the $e-h$ system. In this situation, the crossover problem $13,14,15,16$ between the BEC of excitons and the $e-h$ BCSlike state is fascinating, especially from the viewpoint of the difference from the BCS-BEC crossover in superconductors or trapped atomic Fermi gases 17.18 .19 .20 We believe that revealing such differences will deepen our understanding of $e-h$ systems, leading toward the experimental observation. Compared with other systems undergoing condensation of bound pairs, electrons and holes in a quasi-thermal-equilibrium system in semiconductors have the following two notable characteristics: (i) They involve not only the $e-h$ attractive Coulomb interaction but also the repulsive one between like particles (besides the Pauli exclusion principle), and (ii) they generally have different masses and mass anisotropies. To understand the coherent condensation of $e-h$ pairs, therefore, we highlight these important characteristics in this work. Early and quite recent works ${ }^{21,22,23}$ based only on the BCS-like meanfield theory have shown that the mass difference and the mass anisotropy suppress the $e-h \mathrm{BCS}$ order. In the presence of such asymmetry, however, it is still unclear how the crossover from the $e-h$ BCS state to the excitonic BEC evolves. In addition, little attention has been paid to the roles of electron-electron $(e-e)$ and hole-hole $(h-h)$ repulsive interactions in this problem.

In this paper, by calculating the transition temperature $T_{c}$ that directly reflects the excitonic BCS-BEC crossover, we clarify the effects of the repulsive interaction and the mass difference on the $e-h$ pair condensation from the BCS to the BEC regime. A simple two-band Hubbard model with attractive and repulsive on-site interactions is adopted to describe the $e-h$ systems. Here we suppose that conduction electrons and valence holes, whose bands are isotropic, have infinite lifetime, and the number of electrons is equal to that of holes $\left(N_{e}=N_{h}\right)$. In our model the interaction strengths and the $e-h$ density are treated as independent parameters. Several physical quantities, such as the density of states, the density of occupied sites, and the quasiparticle weights, are calculated to discuss the properties of the normal phase.

We employ the self-consistent $t$-matrix approximation (SCTMA) in our analysis. The SCTMA is an effective method for the BCS-BEC crossover problem at finite temperatures, ${ }^{24.25 .26}$ and is a conserving approximation based on the Baym-Kadanoff theory. This approximation deals correctly with two-particle correlations. Thus it becomes asymp- 
totically exact in the low-density limit. In addition to the SCTMA, we also use a local approximation (LA), which is justified in high spatial dimensions. The procedure is to neglect the momentum dependence of the self-energy and the vertex function. ${ }^{26}$ It is in the same spirit as the $\mathbf{k}$-averaged approximation ${ }^{27}$ or the dynamical mean-field theory (DMFT) ${ }^{28}$ As known in the DMFT literature, the LA itself (without other approximations) becomes exact in the limit of infinite spatial dimensions and a good approximation for three-dimensional systems. Although the SCTMA combined with the LA is not exact even in the infinite-coordination limit since only ladder diagrams are summed up, it has been successful for the superconductivity of the single-band attractive Hubbard model in high dimensions ${ }^{26}$ In particular, the successive interpolation between the BCS limit with $T_{c} \propto \exp \left(t / U^{\prime}\right)$ and the BEC limit with $T_{c} \propto t^{2} / U^{\prime}$ can be described well, ${ }^{17,18}$ where $U^{\prime}$ and $t$ denote the attractive interaction and the transfer energy, respectively. Therefore, we extend the scheme to our twoband model, and expect that our results are valid for threedimensional bulk systems.

This paper is organized as follows. In Sec. II, the SCTMA combined with the LA is applied to the normal phase for the $e-h$ two-band Hubbard model. In Sec. III, several physical quantities are calculated and properties of the normal phase are discussed. In Sec. IV, the transition temperature from the normal phase to the $e-h$ pair condensed phase is presented as a function of the $e-h$ attraction strength for various parameters. We examine in Sec. IV A the effect of the $e-e(h-h)$ repulsion on the transition temperature. In Sec. IV B, the effect of the mass difference on the transition temperature is analyzed and features of the BCS-BEC crossover in the $e-h$ system are discussed. Concluding remarks are given in Sec. V.

\section{FORMULATION}

We consider an $e-h$ system described by the two-band Hubbard model. The Hamiltonian is given by

$$
\begin{aligned}
H= & -\sum_{\langle i j\rangle, \sigma} \sum_{\alpha=e, h} t_{\alpha} c_{i \sigma}^{\alpha \dagger} c_{j \sigma}^{\alpha}-\sum_{j \sigma, \alpha} \mu_{\alpha} n_{j \sigma}^{\alpha} \\
& +U \sum_{j, \alpha} n_{j \uparrow}^{\alpha} n_{j \downarrow}^{\alpha}-U^{\prime} \sum_{j \sigma \sigma^{\prime}} n_{j \sigma}^{e} n_{j \sigma^{\prime}}^{h},
\end{aligned}
$$

where $c_{j \sigma}^{e \dagger}\left(c_{j \sigma}^{h \dagger}\right)$ denotes a creation operator of an electron (a hole) with spin $\sigma=\{\uparrow, \downarrow\}$ at the $j$ th site and $n_{j \sigma}^{\alpha}=c_{j \sigma}^{\alpha \dagger} c_{j \sigma}^{\alpha}$ with $\alpha=\{e, h\}$. The quantities $t_{e}\left(t_{h}\right)$ and $\mu_{e}\left(\mu_{h}\right)$ are the transfer integral of the electrons (holes) between the nearestneighbor sites and the chemical potential measured from the center of the bare electron (hole) band, respectively. The onsite Coulomb interaction of the $e-e(h-h)$ repulsion and that of the $e-h$ attraction are expressed by $U$ and $-U^{\prime}$, respectively.

We apply the SCTMA to the model (11). Feynman diagrams contributing to the self-energy of electrons and holes in the normal phase within the SCTMA are shown in Fig. 1, where all particle-hole (particle-particle) ladder diagrams are taken into account with respect to the interaction $U\left(-U^{\prime}\right)$. The ex-



FIG. 1: Feynman diagrams for the self-energy in the normal phase. The solid line denotes the electron or hole Green's function $G_{\alpha}$ where $\bar{\alpha}=h(e)$ for $\alpha=e(h)$. The spin weight is 2 for the diagram of the vertex function $\Gamma_{\alpha \bar{\alpha}}$.

plicit expression of the self-energy is given by

$$
\begin{aligned}
\Sigma_{\alpha}\left(\mathbf{k}, \omega_{n}\right)= & \frac{T}{N} \sum_{\mathbf{q}, v_{m}} e^{i\left(v_{m}+\omega_{n}\right) 0^{+}} \Gamma_{\alpha \alpha}\left(\mathbf{q}, v_{m}\right) \\
& \times G_{\alpha}\left(\mathbf{q}+\mathbf{k}, v_{m}+\omega_{n}\right) \\
& +\frac{2 T}{N} \sum_{\mathbf{q}, v_{m}} e^{i\left(v_{m}-\omega_{n}\right) 0^{+}} \Gamma_{\alpha \bar{\alpha}}\left(\mathbf{q}, v_{m}\right) \\
& \times G_{\bar{\alpha}}\left(\mathbf{q}-\mathbf{k}, v_{m}-\omega_{n}\right),
\end{aligned}
$$

where $N$ is the total number of lattice sites, $T$ denotes the temperature, $\omega_{n}=(2 n+1) \pi T$, and $v_{m}=2 \pi m T$ with integer $n$ and $m$. The symbol $0^{+}$in the convergence factor denotes a time infinitesimally later than $\tau=0$. Here the spin index $\sigma$ is omitted because we now consider the spin-symmetric case, but the spin weight (a factor 2) is counted. The single-particle Matsubara Green's function $G_{\alpha}\left(\mathbf{k}, \omega_{n}\right)$ defined by the Fourier transform of $-\left\langle T_{\tau} c_{j \sigma}^{\alpha}(\tau) c_{j^{\prime} \sigma}^{\alpha \dagger}\right\rangle$ is expressed in terms of the selfenergy (2) as

$$
G_{\alpha}\left(\mathbf{k}, \omega_{n}\right)=\frac{1}{i \omega_{n}+\mu_{\alpha}-\varepsilon_{\mathbf{k}}^{\alpha}-\Sigma_{\alpha}\left(\mathbf{k}, \omega_{n}\right)},
$$

where $\varepsilon_{\mathrm{k}}^{\alpha}$ is the band dispersion of the noninteracting electrons/holes. The two-particle vertex functions $\Gamma_{\alpha \alpha^{\prime}}\left(\mathbf{q}, v_{m}\right)$ are obtained as the infinite geometric series,

$$
\begin{aligned}
\Gamma_{\alpha \alpha}\left(\mathbf{q}, v_{m}\right) & =U+U K_{\alpha \alpha}\left(\mathbf{q}, v_{m}\right) U+\cdots \\
& =\frac{U}{1-U K_{\alpha \alpha}\left(\mathbf{q}, v_{m}\right)}, \\
\Gamma_{\alpha \bar{\alpha}}\left(\mathbf{q}, v_{m}\right) & =-U^{\prime}+\left(-U^{\prime}\right) K_{\alpha \bar{\alpha}}\left(\mathbf{q}, v_{m}\right)\left(-U^{\prime}\right)+\cdots \\
& =\frac{-U^{\prime}}{1+U^{\prime} K_{\alpha \bar{\alpha}}\left(\mathbf{q}, v_{m}\right)}
\end{aligned}
$$

where the pair propagators are

$$
\begin{aligned}
K_{\alpha \alpha}\left(\mathbf{q}, v_{m}\right) & =-\frac{T}{N} \sum_{\mathbf{k}, \omega_{n}} G_{\alpha}\left(\mathbf{k}, \omega_{n}\right) G_{\alpha}\left(\mathbf{q}+\mathbf{k}, v_{m}+\omega_{n}\right), \\
K_{\alpha \bar{\alpha}}\left(\mathbf{q}, v_{m}\right) & =-\frac{T}{N} \sum_{\mathbf{k}, \omega_{n}} G_{\alpha}\left(\mathbf{k}, \omega_{n}\right) G_{\bar{\alpha}}\left(\mathbf{q}-\mathbf{k}, v_{m}-\omega_{n}\right) .
\end{aligned}
$$


By using the self-consistent set of Eqs. (2)-(7), the model (1) can be solved within the SCTMA. However, a straightforward calculation is very difficult especially for three-dimensional systems with large system size $N$. In this work, then, we combine a LA with the SCTMA to simplify the above self-consistent calculation. ${ }^{26,27}$ The procedure is performed by neglecting momentum dependence of the selfenergy and the vertex function [i.e., $\Sigma_{\alpha}\left(\mathbf{k}, \omega_{n}\right) \rightarrow \Sigma_{\alpha}\left(\omega_{n}\right)$ and $\left.\Gamma_{\alpha \alpha^{\prime}}\left(\mathbf{q}, v_{m}\right) \rightarrow \Gamma_{\alpha \alpha^{\prime}}\left(v_{m}\right)\right]$. This analysis is equivalent to solving an effective single-impurity problem of the DMFT within the SCTMA. By making the LA, the self-consistent Eqs. (2)(7) can be written in terms of only local functions:

$$
\begin{aligned}
\Sigma_{\alpha}\left(\omega_{n}\right)= & T \sum_{v_{m}} e^{i\left(v_{m}+\omega_{n}\right) 0^{+}} \Gamma_{\alpha \alpha}\left(v_{m}\right) G_{\alpha}\left(v_{m}+\omega_{n}\right) \\
& +2 T \sum_{v_{m}} e^{i\left(v_{m}-\omega_{n}\right) 0^{+}} \Gamma_{\alpha \bar{\alpha}}\left(v_{m}\right) G_{\bar{\alpha}}\left(v_{m}-\omega_{n}\right)
\end{aligned}
$$

where the local Green's function is

$$
\begin{aligned}
G_{\alpha}\left(\omega_{n}\right) & =\frac{1}{N} \sum_{\mathbf{k}} G_{\alpha}\left(\mathbf{k}, \omega_{n}\right) \\
& =\int d \varepsilon \frac{\rho_{\alpha}^{0}(\varepsilon)}{i \omega_{n}+\mu_{\alpha}-\varepsilon-\Sigma_{\alpha}\left(\omega_{n}\right)},
\end{aligned}
$$

with the noninteracting density of states $\rho_{\alpha}^{0}(\varepsilon)$, and the local vertex functions are

$$
\begin{aligned}
& \Gamma_{\alpha \alpha}\left(v_{m}\right)=\frac{U}{1-U K_{\alpha \alpha}\left(v_{m}\right)}, \\
& \Gamma_{\alpha \bar{\alpha}}\left(v_{m}\right)=\frac{-U^{\prime}}{1+U^{\prime} K_{\alpha \bar{\alpha}}\left(v_{m}\right)},
\end{aligned}
$$

with the local pair propagators

$$
\begin{aligned}
& K_{\alpha \alpha}\left(v_{m}\right)=-T \sum_{\omega_{n}} G_{\alpha}\left(\omega_{n}\right) G_{\alpha}\left(v_{m}+\omega_{n}\right), \\
& K_{\alpha \bar{\alpha}}\left(v_{m}\right)=-T \sum_{\omega_{n}} G_{\alpha}\left(\omega_{n}\right) G_{\bar{\alpha}}\left(v_{m}-\omega_{n}\right) .
\end{aligned}
$$

To determine the transition temperature of the condensation of $e-h$ pairs, we examine the $e-h$ singlet pair susceptibility $^{29} \chi\left(\mathbf{q}, v_{m}\right)$, defined by the Fourier transform of $-\left\langle T_{\tau} c_{j,-\sigma}^{h}(\tau) c_{j \sigma}^{e}(\tau) c_{j^{\prime} \sigma}^{e^{\dagger}}\left(\tau^{\prime}\right) c_{j^{\prime},-\sigma}^{h \dagger}\left(\tau^{\prime}\right)\right\rangle$, which is given by the ladder terms corresponding to $\Gamma_{e h}\left(\mathbf{q}, v_{m}\right)$, i.e.,

$$
\chi\left(\mathbf{q}, v_{m}\right)=\frac{K_{e h}\left(\mathbf{q}, v_{m}\right)}{1+U^{\prime} K_{e h}\left(\mathbf{q}, v_{m}\right)} .
$$

Here note that within the present SCTMA the repulsive interaction $U$ does not appear explicitly in Eq. (14), but it influences the $e$ - $h$ pair susceptibility through the single-particle Green's functions in Eq. (7). If the uniform static $e-h$ pair susceptibility $\chi(\mathbf{0}, 0)$ diverges for $T \searrow T_{c}$, it is a signal of the onset of the $e$ - $h$ pair condensation (the Thouless criterion). Thus the transition temperature $T_{c}$ can be determined as the temperature satisfying the condition

$$
1+U^{\prime} K_{e h}(\mathbf{0}, 0)=0 .
$$

Since the self-energy is local (i.e., momentum independent), the pair propagator $K_{e h}(\mathbf{0}, 0)$ in Eq. 15 can be expressed in terms of only local functions as

$$
\begin{aligned}
K_{e h}(\mathbf{0}, 0) & =-\frac{T}{N} \sum_{\mathbf{k}, \omega_{n}} G_{e}\left(\mathbf{k}, \omega_{n}\right) G_{h}\left(-\mathbf{k},-\omega_{n}\right) \\
& =-T \sum_{\omega_{n}} \frac{G_{e}\left(\omega_{n}\right)-\gamma G_{h}\left(\omega_{n}\right)^{*}}{\zeta_{h}\left(\omega_{n}\right)^{*}-\gamma \zeta_{e}\left(\omega_{n}\right)},
\end{aligned}
$$

where $\zeta_{\alpha}\left(\omega_{n}\right)=i \omega_{n}+\mu_{\alpha}-\Sigma_{\alpha}\left(\omega_{n}\right)$ and $\gamma=t_{h} / t_{e}$ is defined as the $e$ - $h$ band mass ratio $m_{e} / m_{h}$. Here we have assumed the relation $\varepsilon_{\mathbf{k}}^{h}=\gamma \varepsilon_{\mathbf{k}}^{e}$. In this paper we use $\rho_{\alpha}^{0}(\varepsilon)=$ $\sqrt{4 t_{\alpha}^{2}-\varepsilon^{2}} /\left(2 \pi t_{\alpha}^{2}\right)$ as a typical density of states of threedimensional systems.

\section{PROPERTIES OF NORMAL PHASE}

In this section, properties of the normal phase above $T_{c}$ are discussed by using the self-consistent solution of Eqs. 8 13. We analyze the interacting density of states, the density of sites occupied by electrons and holes, and the quasiparticle weight, for various values of $U, U^{\prime}, \gamma=t_{h} / t_{e}$, and the particle density $n$. In the following we consider the cases of $n=0.25$ and 0.5 (and $n=1$ only in the next section), where the particle density is defined as

$$
n \equiv \sum_{\sigma}\left\langle n_{j \sigma}^{\alpha}\right\rangle=2 T \sum_{\omega_{n}} e^{i \omega_{n} 0^{+}} G_{\alpha}\left(\omega_{n}\right)
$$

under the condition of charge neutrality $\left\langle n_{\sigma}^{e}\right\rangle=\left\langle n_{\sigma}^{h}\right\rangle$. Throughout the paper the quantity $t_{e}+t_{h}$ is taken as the energy unit.

\section{A. Density of states}

Figure 2 shows the interacting density of states per spin,

$$
\rho_{\alpha}(\omega)=-\frac{1}{\pi} \operatorname{Im} G_{\alpha}\left(\omega+i 0^{+}\right),
$$

for various values of $U^{\prime}$. Parameters are chosen as $n=0.25$ (1/8 filling), $T=0.04$ (that is, above $T_{c}$ ), and $\gamma=1$ with the fixed $U=$ (a) 0 and (b) 2 . Note that $\rho_{e}(\omega)=\rho_{h}(\omega)$ for $\gamma=1$. Here we have obtained the retarded Green's function $G_{\alpha}(\omega+$ $i 0^{+}$) from the numerical analytic continuation by using the Padé approximation.

When $U=0, \rho_{\alpha}(\omega)$ exhibits a characteristic two-peak structure as $U^{\prime}$ is increased: a sharp peak (i.e., the quasiparticle coherent peak) appears at $\omega \simeq 0$ and its weight decreases, while a broad incoherent peak develops at $\omega \simeq U^{\prime}$. We should remark that in the low-density limit $(n \rightarrow 0)$ an $e-h$ bound state is formed for $U^{\prime} \geq 1$ and the binding energy is given by $E_{b} \simeq U^{\prime}$. Hence, such a behavior of the density of states implies that local $e$ - $h$ pairs (excitons) tend to be formed, which will become clear also from the analysis of the density of occupied sites (Fig. 3). 


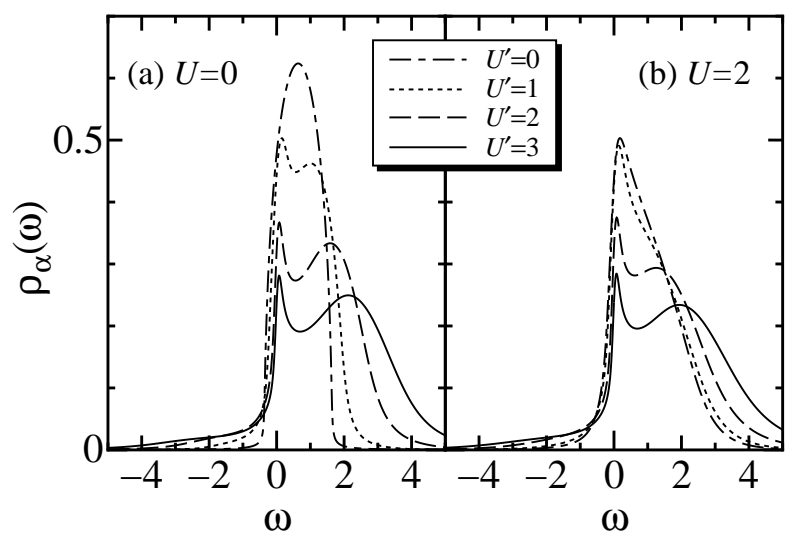

FIG. 2: The density of states $\rho_{\alpha}(\omega)$ for $n=0.25, T=0.04$, and $\gamma=t_{h} / t_{e}=1$. All the energies are scaled by $t_{e}+t_{h}$.

When $U=2, \rho_{\alpha}(\omega)$ is broadened by $U$, so it is already strongly renormalized even for $U^{\prime}=0$. This indicates that $U$ gives the dominant contribution to the band gap renormalization. For $U^{\prime}<U\left(U^{\prime}=1\right)$, in contrast to the case of $U=0$, the broad incoherent peak is not observed. This means that the excitonic correlation is suppressed by $U$. For $U^{\prime} \gtrsim U\left(U^{\prime}=2\right.$ and 3$), \rho_{\alpha}(\omega)$ shows the two-peak structure again. Compared with the case of $U=0$, however, the coherent (incoherent) peak is enhanced (suppressed) especially for $U \simeq U^{\prime}$. This is due to the competition between $U$ and $U^{\prime}$. Similar behavior has been found more prominently in the DMFT for $n=1$ (half filling) 30,31 There, the metallic phase is stabilized for $U \simeq U^{\prime} \leq 5$ between the Mott-Hubbard and biexcitonlike insulating phases. Except in such a case, the density of states in Fig.2] suggests fundamentally that the metallic character tends to be lost because of both the weight shifting due to $U^{\prime}$ and the overall broadening due to $U$.

Here we note that within the conserving approximation such as the present SCTMA, the system never becomes an insulator $\left[\rho_{\alpha}(0)=0\right]$ with no broken symmetry for any values of $U$ and $U^{\prime}$. This is a well-known failure of this scheme. ${ }^{26,32}$

\section{B. Density of occupied sites}

Examining the density of occupied sites is an effective way to see how the excitonic correlation increases in the normal phase. Now we focus on the density of sites quadruply occupied by electrons and holes, $D_{e h} \equiv \sum_{\sigma \sigma^{\prime}}\left\langle n_{j \sigma}^{e} n_{j \sigma^{\prime}}^{h}\right\rangle$. This is local susceptibility at equal times and can be written in terms of the local pair propagator 13 :

$$
D_{e h}=\sum_{\sigma \sigma^{\prime}}\left\langle n_{j \sigma}^{e} n_{j \sigma^{\prime}}^{h}\right\rangle=-T \sum_{v_{m}} e^{i v_{m} 0^{+}} \frac{4 K_{e h}\left(v_{m}\right)}{1+U^{\prime} K_{e h}\left(v_{m}\right)} .
$$

We first note the limiting cases. For the noninteracting case $D_{e h}=n^{2}$. If all electrons and holes are perfectly bound as local $e-h$ pairs by $U^{\prime}$, the density of occupied sites is expected to become $D_{e h}=n$ for $U>U^{\prime}$ and $D_{e h}=2 n$ for $U<U^{\prime}$. The former case corresponds to an exciton phase, and the latter

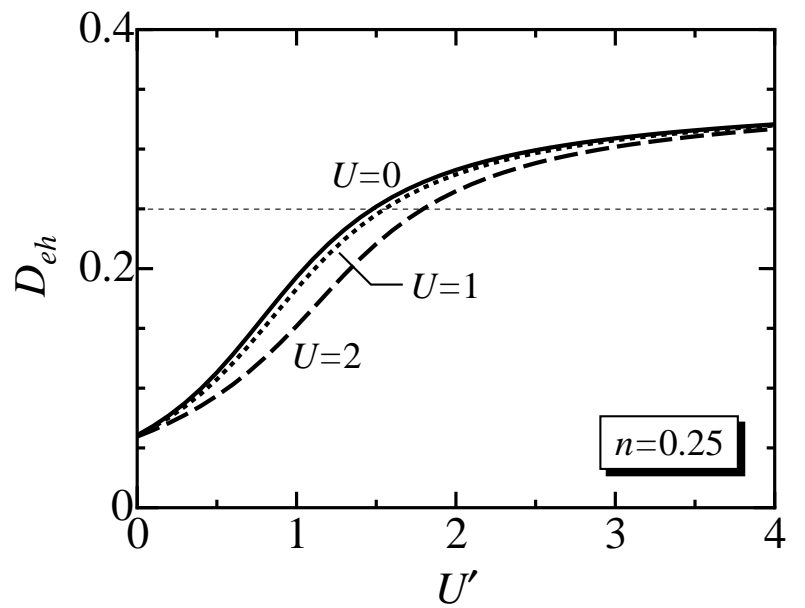

FIG. 3: The density of sites quadruply occupied by electrons and holes as a function of $U^{\prime}$ for $n=0.25, T=0.04$, and $\gamma=1$ (these parameters are the same as in Fig. 2] with fixed $U=0,1$, and 2.

to a biexciton phase where two electrons and two holes (i.e., two excitons) gather on a site. Actually, exciton and biexciton insulating phases with the aforementioned features have been obtained by using DMFT combined with the exact diagonalization method. 31.33

In Fig. 3 the $U^{\prime}$ dependence of $D_{e h}$ is shown for $n=0.25$, $T=0.04$, and $\gamma=1$ (corresponding to Fig. 2) with fixed $U=0,1$, and 2 . For $U^{\prime}=0$, the density of occupied sites becomes $D_{e h} \simeq n^{2}=0.0625$, that is the value in the noninteracting case, regardless the value of $U$. With increasing $U^{\prime}$, $D_{e h}$ increases monotonically. This tendency clearly indicates the development of the excitonic correlation toward the formation of local $e-h$ pairs. To detect trends more evidently, we drew the thin dotted line $\left(D_{e h}=n=0.25\right)$ as a loose guide for a criterion of local $e-h$ pair formation. Here, of course, we should keep in mind that the system is as metallic as ever, as seen in Fig. 2 even if $D_{e h}$ is above the line. One can find that the excitonic correlation is suppressed by $U$ for $U \simeq U^{\prime}$. In the large- $U^{\prime}$ region where the effect of $U$ is suppressed, $D_{e h}$ increases beyond 0.25 as a result of the enhancement of the biexcitonic correlation. However, $D_{e h}$ does not reach $2 n=0.5$ corresponding to the biexciton phase. This is because the $t$ matrix approximation cannot deal adequately with the fourbody correlation, irrespective of the LA. Hence problems of biexcitons and their condensation are out of the scope of this paper.

\section{Renormalization factor}

Here we consider the case that the electron and hole band masses are different $(\gamma \neq 1)$. Evaluating the renormalization factor, we examine the properties of the normal phase in the case with mass difference. We define the renormalization fac- 




FIG. 4: The renormalization factor $Z_{\alpha}$ as a function of $U$ for $n=0.5$, $T=0.05$, and $\gamma=0.5$ with fixed $U^{\prime}=0,1$, and 2 . The solid (dashed) curve denotes $Z_{e}\left(Z_{h}\right)$.

tor $Z_{\alpha}$ by

$$
\begin{aligned}
Z_{\alpha}^{-1} & =1-\left.\frac{d \Sigma_{\alpha}\left(\omega_{n}\right)}{d\left(i \omega_{n}\right)}\right|_{i \omega_{n} \rightarrow 0} \\
& \simeq 1-\frac{\operatorname{Im}\left[\Sigma_{\alpha}(i \pi T)-\Sigma_{\alpha}(-i \pi T)\right]}{2 \pi T} .
\end{aligned}
$$

The quantity $Z_{\alpha}$ at $T \rightarrow 0$ has generally several physical meanings characterizing the Fermi liquid phase, e.g., the weight of the quasiparticle coherent peak in the density of states and the jump at the Fermi wave number in the momentum distribution function. Since the self-energy is momentum independent, it also gives directly the effective mass enhancement of quasiparticles, $m_{\alpha} / m_{\alpha}^{*}$.

In Fig. 4 we show the $U$ dependence of $Z_{\alpha}$ for $n=0.5$ (quarter filling), $T=0.05$, and $\gamma=0.5$ (the hole band mass is twice that of the electron) with fixed $U^{\prime}=0,1$, and 2. For $U^{\prime}=0$, both $Z_{e}$ and $Z_{h}$ are monotonically decreasing functions with respect to $U$. Because of the narrow hole band, the effective repulsive interaction between holes $U /\left(2 t_{h}\right)$ is quite strong, leading to a large reduction of $Z_{h}$ (dashed curve). The presence of $U^{\prime}\left(U^{\prime}=1\right.$ and 2) reduces both $Z_{e}$ and $Z_{h}$. However, the behaviors of $Z_{e}$ and $Z_{h}$ for $U^{\prime} \neq 0$ are qualitatively different from those for $U^{\prime}=0: Z_{h}$ decreases slowly and monotonically with increasing $U$, while $Z_{e}$ has a slight hump (solid curve). The origin of this hump is the competition between the effective repulsion $U /\left(2 t_{e}\right)$ and the effective $e$ - $h$ attraction $U^{\prime} /\left(t_{e}+t_{h}\right)$, which raises the mobility of electrons. When $\gamma=0.5$, therefore, the hump of $Z_{e}$ appears at $U \simeq 2 t_{e} /\left(t_{e}+t_{h}\right) \times U^{\prime}=2 U^{\prime} /(1+\gamma) \simeq 1.3$ and 2.7 for $U^{\prime}=1$ and 2 , respectively. For holes the influence of the competi-

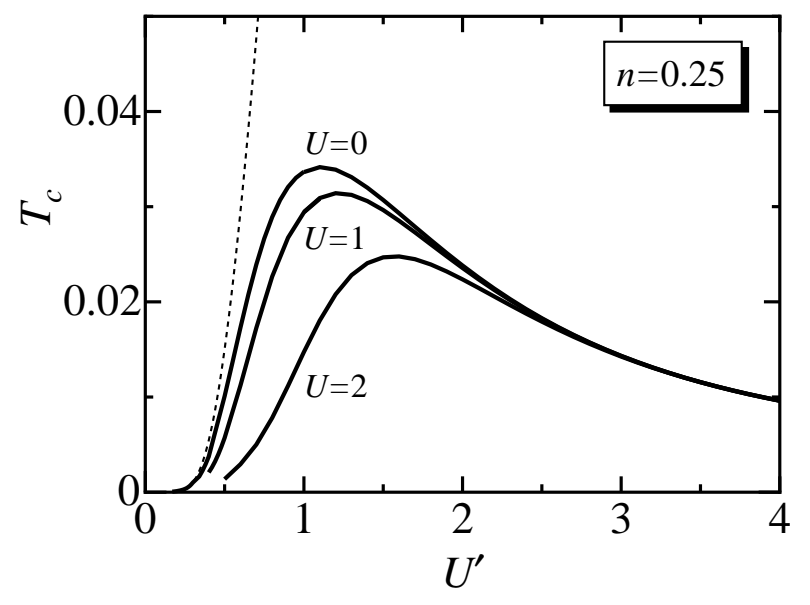

FIG. 5: The transition temperature $T_{c}$ as a function of $U^{\prime}$ for $n=0.25$ and $\gamma=1$ with fixed $U=0,1$, and 2 . The dotted line denotes the result from BCS theory.

tion between $U /\left(2 t_{h}\right)$ and $U^{\prime} /\left(t_{e}+t_{h}\right)$ is quite weak, and $Z_{h}$ is almost constant in the region of $U \lesssim 2 \gamma U^{\prime} /(1+\gamma) \simeq 0.7$ and 1.3 for $U^{\prime}=1$ and 2, respectively. Here we point out that $Z_{\alpha}$ becomes insensitive to $U$ for $U^{\prime}=2$. When $U^{\prime}=2$, the tendency to form bound $e$ - $h$ pairs begins to grow strongly, indicating very weak $U$ dependence. This fact will also appear in the behavior of the transition temperature discussed in the next section.

\section{TRANSITION TEMPERATURE}

In this section, based on the self-consistent solution and the condition defined in 15 , we evaluate the transition temperature $T_{c}$ from the normal phase to the $e-h$ pair condensed phase. By analyzing the $U^{\prime}$ dependence of $T_{c}$ for various $U$ and $\gamma$, the effects of the repulsive interaction and the mass difference on the transition temperature are discussed.

\section{A. Effect of $U$}

First, we consider the effect of the repulsive interaction $U$ on the transition temperature $T_{c}$. The calculations in this subsection are restricted to the mass ratio $\gamma=1$.

Figure 5 shows the $U^{\prime}$ dependence of $T_{c}$ for $n=0.25$ and $\gamma=1$ with fixed $U=0,1$, and 2 . The $e$ - $h$ pair susceptibility in the normal phase diverges for $T \searrow T_{c}$, which means that the $e-h$ pair condensed phase is realized for $T<T_{c}$. For $U=0, T_{c}$ can be described well by the BCS result (dotted curve) in the weak-coupling region $\left(U^{\prime} \lesssim 0.3\right)$, that is, $T_{c} \propto \exp \left(-A / U^{\prime}\right)$ with a constant $A$. With increasing $U^{\prime}$, the solid curve deviates from the BCS result, reaches a maximum at $U^{\prime} \simeq 1$, and then decreases as $1 / U^{\prime}$ for large $U^{\prime}$. The $U^{\prime}$ dependence of $T_{c}$ for large $U^{\prime}$ is related to the behavior of the BEC temperature of a lattice boson system ${ }^{17,18,26}$ with kinetic and potential energies of order $1 / U^{\prime}, 34$ Obviously this result implies 


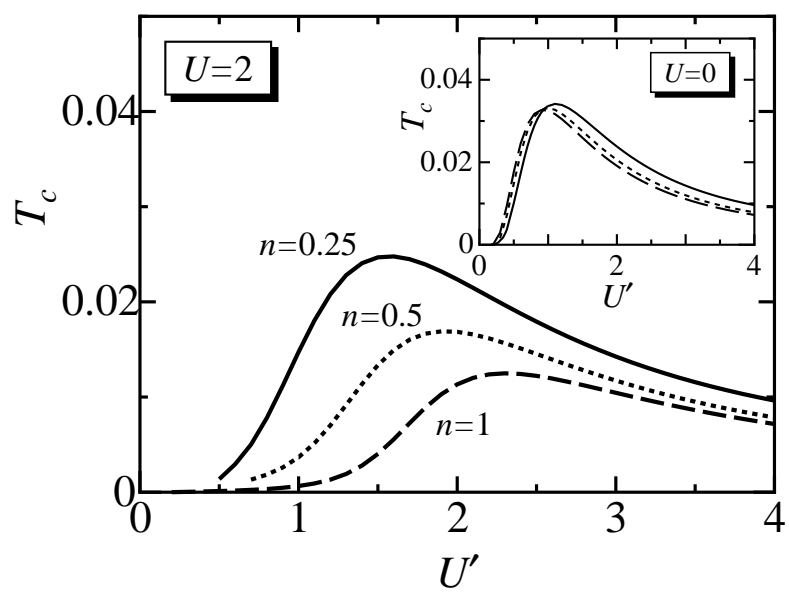

FIG. 6: The transition temperature $T_{c}$ as a function of $U^{\prime}$ for $\gamma=1$ and $U=2$ with fixed $n=0.25,0.5$, and 1.0. The inset shows the case of $U=0$ for $n=0.25$ (solid line), 0.5 (dotted line), and 1.0 (dashed line).

the BCS-BEC crossover, as expected. Note that the ladder terms for the dressed Green's function is essential to extract the above successive crossover ${ }^{24}$ In the presence of $U(U=1$ and 2), one can see the reduction of $T_{c}$ for $U^{\prime} \lesssim U$. This fact can be understood as a consequence of the suppression of the excitonic correlation discussed in Figs. 2 and 3 In contrast, $T_{c}$ is largely insensitive to the repulsion $U$ for $U^{\prime} \gtrsim U$. This supports the validity of physical picture of strongly bound $e-h$ pairs that behave almost like neutral bosons for $U^{\prime} \gtrsim U$, which is consistent with the analysis of the density of occupied sites in Fig. 3

Next, the effect of the repulsion $U$ on the transition temperature is examined by changing the $e-h$ particle density. In Fig. 6 the $U^{\prime}$ dependence of $T_{c}$ with $\gamma=1$ and $U=2$ is shown for $n=0.25,0.5$, and 1 (half filling). The inset displays the corresponding $U^{\prime}$ dependence of $T_{c}$ for $U=0$. When $U=0$ (inset of Fig. 6), the transition temperature $T_{c}$ increases slightly in the weak-coupling region $\left(U^{\prime} \ll 1\right)$ as the particle density $n$ is increased. This behavior is quite reasonable for $U^{\prime} \ll 1$ from the following reason. Within the BCS theory the effective attractive interaction between electrons and holes is roughly given by $\rho_{\alpha}^{0}\left(\varepsilon_{F}^{\alpha}\right) U^{\prime}$ where $\varepsilon_{F}^{\alpha}$ is the Fermi energy for an uncorrelated system [see Eq. [21)]. Hence the effective attraction reaches maximum when $n=1$, i.e., the band is half filled, in the case of the semicircular density of states. The presence of $U$, however, completely changes this tendency. As seen in the main figure of Fig. $6(U=2), T_{c}$ is sufficiently suppressed by $U$ for $U^{\prime} \lesssim U$ as $n$ approaches 1 . When we simply consider the effect of $U$ in the BCS regime, the effective interaction $\rho_{\alpha}^{0}\left(\varepsilon_{F}^{\alpha}\right) U^{\prime}$ could be replaced by $\rho_{\alpha}\left(\varepsilon_{F}^{\alpha}\right) U^{\prime}$, where $\rho_{\alpha}\left(\varepsilon_{F}^{\alpha}\right)$ is strongly renormalized by $U$, as seen in Fig. 2 The renormalization becomes strong as $n$ approaches $1.32 \mathrm{As}$ a result, the effective interaction is reduced by $U$ as $n \rightarrow 1$, leading to the suppression of $T_{c}$. Meanwhile, for large $U^{\prime}$ the contribution of $U$ to $T_{C}$ is very small regardless of the value of $n$.

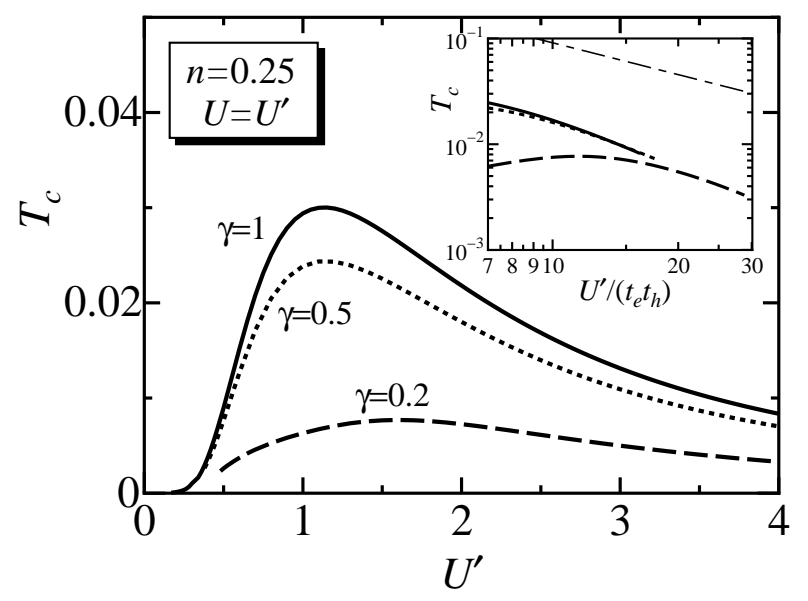

FIG. 7: The transition temperature $T_{c}$ as a function of $U^{\prime}$ for $n=0.25$ and $U=U^{\prime}$ with fixed $\gamma=1,0.5$, and 0.2 . In the inset, $T_{c}$ is shown as a function of $U^{\prime} /\left(t_{e} t_{h}\right)$ for $\gamma=1$ (solid line), 0.5 (dotted line), and 0.2 (dashed line), where the decimal logarithm is taken for both axes. The dot-dashed line represents the exact result (22) in the limit of $U \rightarrow \infty$ and large $U^{\prime}$.

\section{B. Effect of mass difference}

Finally, we investigate the effect of the mass difference $\gamma$ on the transition temperature $T_{c}$.

We plot the $U^{\prime}$ dependence of $T_{c}$ for $n=0.25$ and $U=U^{\prime}$ in Fig. 7 with fixed $\gamma=1,0.5$, and 0.2. The mass difference has the effect of reducing the transition temperature, which is qualitatively consistent with the result of the BCS-like pairing theory by Mizoo et $a l^{23}$ In addition to the reduction of $T_{c}$ in the weak-coupling BCS regime, our result also involves the suppression of $T_{c}$ in the strong-coupling BEC regime. Such a behavior of $T_{c}$ can be understood analytically in the weakand strong-coupling limits as follows.

In the weak-coupling limit, the transition temperature can be estimated by using the Green's function including only the Hartree term in its own self-energy, which is given by

$$
T_{c}^{\mathrm{BCS}}=1.13 \sqrt{w_{c}^{e} w_{c}^{h}} \exp \left(-\frac{t_{e}+t_{h}}{2 t_{\alpha} \rho_{\alpha}^{0}\left(\varepsilon_{F}^{\alpha}\right) U^{\prime}}\right),
$$

where $w_{c}^{\alpha}$ is a cutoff energy of order $\varepsilon_{F}^{\alpha}$. Note that $t_{e} \rho_{e}^{0}\left(\varepsilon_{F}^{e}\right)=$ $t_{h} \rho_{h}^{0}\left(\varepsilon_{F}^{h}\right)$. Equation 21] indicates that the effective $e$ - $h$ attractive interaction is given by $t_{\alpha} \rho_{\alpha}^{0}\left(\varepsilon_{F}^{\alpha}\right) U^{\prime}$, as mentioned above. Moreover, another important point derived from Eq. 21) is that the system is characterized by an energy scale $t_{e}+t_{h}$ determining the dimensionless effective coupling strength in the weak-coupling region. In our model the quantity $\left(t_{e}+t_{h}\right)^{-1}$ is proportional to $m_{e} m_{h} /\left(m_{e}+m_{h}\right)$, namely, the reduced mass. Therefore, the transition to the $e-h$ pair condensed phase in the weak-coupling region is related to the relative motion between electrons and holes, implying the BCS regime. The $\gamma$ dependence of $T_{c}$ in the BCS regime can be roughly evaluated from that of the cutoff energy $w_{c}^{\alpha}$, where we should recall that the argument of the exponential in Eq. (21) does not depend on the mass ratio $\gamma$ when $t_{e}+t_{h}$ is taken as the energy unit. 
The cutoff energy of holes $w_{c}^{h}$ should be $\gamma w_{c}^{e}\left(\sim \gamma t_{e}\right)$ since $\varepsilon_{F}^{h}=\gamma \varepsilon_{F}^{e}\left(\sim \gamma t_{e}\right)$. Thus the coefficient of Eq. (21) becomes $\sqrt{w_{c}^{e} w_{c}^{h}} \sim \sqrt{\gamma} t_{e}$, and so it is found that $T_{c}$ in the BCS regime would be approximately proportional to $\sqrt{\gamma} /(1+\gamma)$.

On the other hand, in the strong-coupling limit, the model (1) can be mapped onto a single-band attractive Hubbard model with the interaction $-U^{\prime}$ and the hopping $t_{\alpha}$ at $U=\infty$. For large $U^{\prime}$, this attractive Hubbard model can map to a hard core Boson model with the kinetic energy $2 t_{e} t_{h} / U^{\prime}$ and the potential energy $\left(t_{e}^{2}+t_{h}^{2}\right) / U^{\prime}$. Using the standard meanfield theory, which becomes exact in the limit of the large coordination number of the lattice, we can obtain the BEC temperature in the limit of $U \rightarrow \infty$ and large $U^{\prime}$ as ${ }^{35}$

$$
T_{c}^{\mathrm{BEC}}=\frac{2 t_{e} t_{h}}{U^{\prime}} \frac{2 n-1}{\ln [n /(1-n)]} .
$$

This expression indicates that the system is characterized by an energy scale $t_{e} t_{h} /\left(t_{e}+t_{h}\right)$ determining the dimensionless effective coupling strength in the strong-coupling region, which is related to the motion of the center of mass since $\left(t_{e}+t_{h}\right) /\left(t_{e} t_{h}\right) \propto m_{e}+m_{h}$. The $U^{\prime}$ dependence of $T_{c}$ in the strong-coupling region of Fig. 7 can be described asymptotically in terms of $t_{e} t_{h} / U^{\prime}$. In the inset of Fig. 7 the transition temperature is shown together with the result of Eq. (22), where $U^{\prime} /\left(t_{e} t_{h}\right)$ is taken as the $x$ axis and the decimal logarithm is taken for both axes. The transition temperature $T_{c}$ for various mass ratio $\gamma$ tends to behave linearly with the slope of -1 (i.e., $T_{c} \propto 1 / U^{\prime}$ ) and merges in the large- $U^{\prime}$ region, implying the BEC regime. As seen from Eq. (22), the $\gamma$ dependence of $T_{c}$ in the BEC regime is given by $T_{c} \propto t_{e} t_{h}=\gamma /(1+\gamma)^{2}$. Therefore, it is confirmed that for the fixed $U^{\prime}$ and $n$ the transition temperature is suppressed by the $e-h$ mass difference in the BEC regime, as well as in the BCS regime.

Viewed in this way, the characteristic energy scale and the $e-h$ mass ratio dependence of the transition temperature become clear in both the BCS and BEC regimes. We especially emphasize the point that the excitonic BCS-BEC crossover can be marked by the change of the characteristic energy scale from $t_{e}+t_{h}$ to $t_{e} t_{h} /\left(t_{e}+t_{h}\right)$, which is not necessarily evident in the standard scenario ${ }^{19}$ of the BCS-BEC crossover.

\section{CONCLUDING REMARKS}

In this paper, by applying the self-consistent $t$-matrix approximation with the LA to the $e-h$ two-band Hubbard model, we have discussed the properties of the normal phase and the transition temperature to the $e-h$ pair condensed phase. In the analysis, the effects of the repulsive interaction between like particles and the mass $e$ - $h$ difference have been given special attention.

From the behavior of the calculated physical quantities, we have found that the development of the excitonic correlation induced by the $e-h$ attractive interaction is suppressed in the presence of the repulsion between like particles and also the mass difference. In particular, it is noteworthy that the competition between the effective interactions $U /\left(2 t_{\alpha}\right)$ and $U^{\prime} /\left(t_{e}+t_{h}\right)$ play the key roles in controlling the formation of excitonic bound pairs in the normal phase. This is a remarkable feature of the two-band $e$ - $h$ system.

The behavior of the transition temperature has shown that the present SCTMA combined with the LA can describe well the excitonic BCS-BEC crossover at finite temperatures. In the $\mathrm{BCS}$ regime of weak $e-h$ attraction, the repulsive interaction sufficiently suppresses the transition temperature for $U>U^{\prime}$. In contrast, it does not affect the transition temperature for $U<U^{\prime}$ in the BEC regime of strong $e-h$ attraction. These results imply that the competition between the effective repulsion and attraction plays an important role in understanding the properties of the $e-h$ pair condensed phase and the crossover behavior also below $T_{c}$. Furthermore, we have found that in the whole BCS-BEC regime the $e$ - $h$ mass difference leads to large suppression of the transition temperature. This analysis in the case of $\gamma \neq 1$ has allowed us to capture the BCS-BEC crossover as the change of the characteristic energy scale from $t_{e}+t_{h}$ to $t_{e} t_{h} /\left(t_{e}+t_{h}\right)$, where the former is related to the relative motion and the latter to the motion of the center of mass.

Let us now discuss limitations of the present SCTMA. The $n$ dependence of $T_{c}$ in the large- $U^{\prime}$ region is opposite to that of the exact result in the limit of $U \rightarrow \infty$ and large $U^{\prime}$ given by Eq. (22). The result of Fig. 6 suggests an decreasing function of $n$ while the exact result is an increasing function of $n$. It is not clear at present why these are not consistent even qualitatively. At least we should remind ourselves that the $t$-matrix approximation is valid for the low-density limit.

Another difficulty is that the SCTMA cannot describe insulating states without any symmetry breaking, ${ }^{26.32}$ such as the exciton and biexciton phases. Here we should remark that the SCTMA for the single-band attractive Hubbard mode126 does not yield the pseudogap behavior (or the pairing transition $^{36,37.38 .39}$ ) as seen in high- $T_{c}$ superconductors. The pseudogap phase in the context of superconductors is deeply related to the exciton phase in $e-h$ systems where incoherent $e-h$ bound pairs (which do not condense but are insulating) are formed. In our model, the DMFT combined with the exact diagonalization method ${ }^{31,33}$ indicates the appearance of exciton and biexciton insulating phases that already show up for $U^{\prime} \simeq 2$. Nevertheless, it is still notable that even within the SCTMA one can see the tendency to form $e-h$ bound pairs above $T_{c}$ by calculating the density of states and the density of occupied sites. However, a unified theory for the exciton Mott transition and the excitonic condensation would require nonperturbative approaches such as the full DMFT (Refs. 3133 , and 39) and Monte Carlo methods ${ }^{40.41}$

The long-range part of the Coulomb interaction was not considered here. It may become crucial particularly in the $B E C$ regime, which motivates future work.

As has been argued in Refs. 14 and 15 , clarifying the BCSBEC crossover problem from the optical response attracts great interest and is important from not only the theoretical but also the experimental aspect. For this purpose, the present SCTMA will be extended to the $e-h$ pair condensed phase below $T_{c}$. The analysis for the properties of the condensed phase and optical response will be reported soon in a forthcoming presentation. ${ }^{42}$ 


\section{Acknowledgments}

The authors would like to thank K. Asano, A. Tsuruta, T. Tohyama, and A. Koga for useful discussions and comments. We also thank P. Huai for a critical reading of this paper. The computation in this work has been done using the facilities of the Supercomputer Center, Institute for Solid State Physics, University of Tokyo. This work has been supported by Core Research for Evolutional Science and Technology (CREST), Japan Science and Technology Agency (JST).
1 S. A. Moskalenko and D. W. Snoke, Bose-Einstein Condensation of Excitons and Biexcitons (Cambridge University Press, Cambridge, U.K., 2000).

2 H. Haug and S. Schmitt-Rink, Prog. Quantum Electron. 9, 3 (1984).

3 R. Zimmermann, Many-Particle Theory of Highly Excited Semiconductors (Teubner, Leipzig, 1988).

4 S. A. Moskalenko, Fiz. Tverd. Tela (Leningrad) 4, 276 (1962) [Sov. Phys. Solid State 4, 199 (1962)].

5 J. M. Blatt, K. W. Böer, and W. Brandt, Phys. Rev. 126, 1691 (1962).

6 L. V. Keldysh and Y. V. Kopaev, Fiz. Tverd. Tela (Leningrad) 6, 2791 (1964) [Sov. Phys. Solid State 6, 2219 (1965)].

7 D. Jérome, T. M. Rice, and W. Kohn, Phys. Rev. 158, 462 (1967).

8 D. W. Snoke, Phys. Status Solidi B 238, 389 (2003).

9 D. W. Snoke, J. P. Wolfe, and A. Mysyrowicz, Phys. Rev. Lett. 64, 2543 (1990); Phys. Rev. B 41, 11171 (1990).

10 J. L. Lin and J. P. Wolfe, Phys. Rev. Lett. 71, 1222 (1993).

11 M. Kuwata-Gonokami, R. Shimano, and A. Mysyrowicz, J. Phys. Soc. Jpn. 71, 1257 (2002).

12 P. P. Vasil'ev, Phys. Status Solidi B 241, 1251 (2004).

13 C. Comte and P. Nozières, J. Phys. (France) 43, 1069 (1982); P. Nozières and C. Comte, ibid. 43, 1083 (1982).

14 T. J. Inagaki, M. Aihara, and A. Takahashi, Solid State Commun. 115, 645 (2000).

15 T. J. Inagaki and M. Aihara, Phys. Rev. B 65, 205204 (2002).

16 P. B. Littlewood, P. R. Eastham, J. M. J. Keeling, F. M. Marchetti, B. D. Simons, and M. H. Szymanska, J. Phys.: Condens. Matter 16, S3597 (2004).

17 P. Nozières and S. Schmitt-Rink, J. Low Temp. Phys. 59, 195 (1985).

18 R. Micnas, J. Ranninger, and S. Robaszkiewicz, Rev. Mod. Phys. 62, 113 (1990).

19 M. Randeria, in Bose-Einstein Condensation, edited by A. Griffin, D. W. Snoke, and S. Stringari (Cambridge University Press, Cambridge, U.K., 1995), p. 355.

20 Y. Ohashi and A. Griffin, Phys. Rev. Lett. 89, 130402 (2002); Phys. Rev. A 67, 033603 (2003).

21 Y. V. Kopaev, Fiz. Tverd. Tela (Leningrad) 8, 223 (1966) [Sov. Phys. Solid State 8, 175 (1966)].

22 J. Zittartz, Phys. Rev. 162, 752 (1967).

23 K. Mizoo, T. J. Inagaki, Y. Ueshima, and M. Aihara, J. Phys. Soc. Jpn. 74, 1745 (2005).
24 R. Frèsard, B. Glaser, and P. Wölfle, J. Phys.: Condens. Matter 4, 8565 (1992).

25 R. Haussmann, Z. Phys. B: Condens. Matter 91, 291 (1993); Phys. Rev. B 49, 12975 (1994).

26 M. Keller, W. Metzner, and U. Schollwöck, Phys. Rev. B 60, 3499 (1999).

27 M. Letz and R. J. Gooding, J. Phys.: Condens. Matter 10, 6931 (1998).

28 A. Georges, G .Kotliar, W. Krauth, and M. J. Rozenberg, Rev. Mod. Phys. 68, 13 (1996).

29 We restrict our consideration to a pure $s$-wave pairing, which is valid for low $e$ - $h$ density or for $U=0$ and $\infty$. Of course, the possibility of the state having other pairing symmetry (such as $d$ wave) is not excluded, especially in the case near half filling and with finite $U$. To examine this, however, the momentum dependence of the self-energy is required.

30 A. Koga, Y. Imai, and N. Kawakami, Phys. Rev. B 66, 165107 (2002).

31 Y. Tomio and T. Ogawa, J. Lumin. 112, 220 (2005).

32 B. Menge and E. Müller-Hartmann, Z. Phys. B: Condens. Matter 82, 237 (1991).

33 Y. Tomio and T. Ogawa, in Proceedings of the International Conference on Low Temperature Physics, Orlando, Florida, 2005, edited by Y. Takano (unpublished).

$34 T_{c} \propto 1 / U^{\prime}$ in the lattice model corresponds to the BEC temperature in the continuum model where $T_{c}$ saturates and does not depend on the interaction strength in the BEC regime; see, for example, Ref. 19.

35 The expression of $T_{c}^{\mathrm{BEC}}$ corresponding to the case of $t_{e}=t_{h}$ is seen in Refs. 18 and 36.

36 M. Keller, W. Metzner, and U. Schollwöck, J. Low Temp. Phys. 126, 961 (2002).

37 R. R. dos Santos, Phys. Rev. B 50, R635 (1994).

38 M. Capone, C. Castellani, and M. Grilli, Phys. Rev. Lett. 88, 126403 (2002).

39 A. Toschi, P. Barone, M. Capone, and C. Castellani, New J. Phys. 7, 7 (2005).

40 S. De Palo, F. Rapisarda, and G. Senatore, Phys. Rev. Lett. 88, 206401 (2002).

41 S. W. Koch, W. Hoyer, M. Kira, and V. S. Filinov, Phys. Status Solidi B 238, 404 (2003).

42 Y. Tomio and T. Ogawa (unpublished). 